\title{
Much native data, little longitudinal information: a model of information for the continuity of care, from prenatal assistance to the emergency in maternities
}

\author{
Sá TQV a, Aguiar RALP ${ }^{\text {b }}$, Reis ZSN c
}

a Federal University of Minas Gerais, Departament of Gynecology and Obstetrics, Faculty of Medicine, Belo Horizonte, Minas Gerais, Brazil. ORCID: 0000-0003-1103919X

${ }^{\mathrm{b}}$ Federal University of Minas Gerais, Departament of Gynecology and Obstetrics, Faculty of Medicine, Belo Horizonte, Minas Gerais, Brazil. ORCID: 0000-0003-24703539

${ }^{c}$ Federal University of Minas Gerais, Departament of Gynecology and Obstetrics, Faculty of Medicine, Belo Horizonte, Minas Gerais, Brazil. ORCID: 0000-0001-63749295

thabatasa@gmail.com, regina.alpa@gmail.com, zilma.medicina@gmail.com

Abstract. Obstetric caring demands a continuous process of information sharing between health professionals. However, the lack of communication between points of assistance has allowed for an accumulation of local data without the benefits of data interoperability. The study's objective is to develop an information model with essential obstetric data to foster the continuity of information. An exploratory research involved discussions of fictitious cases of obstetric emergencies and ninety electronic medical records (EMR) were used to validate the model. The minimum antenatal dataset entries was structured into nine sections, and fifty-six data entries. The development of an information model, based on the standard of interoperability, has the potential to overcome the informality of EMR. 


\section{Introduction}

The timely exchange of health information for the provision of care remains a big challenge, including the current replacement of paper-based clinical records on paper by computerized systems. Information sharing between points in the healthcare network and during the life of a patient can result in remarkable benefits for the continuity of care [1]. However, to achieve these advances, the management of data exchange need to include the adoption of semantic and syntactic standards of information, in addition to technological investments. In this milieu, the interoperability between information systems becomes strictly relevant in order to provide data elements for decision support in care scenarios, which require integrated, comprehensive, and timely information [2].

The Continuity of Care Record (CCR) consists of the organized and transportable core of the dataset with the most relevant information regarding a patient [3]. These records are medical notes about clinical appointments, readily available at the next clinical encounter, supporting clear communication among health professionals [4]. Similarly, the summary of care records comprises a set of health records containing the most relevant clinical data, prepared in a way that is accessible to authorized users, sustained by information technology [5]. In fact, the organization of health assistance according to levels of complexity within the network of care always demands data sharing [6].

Data architecture characterization is critical for achieving interoperability. To be exchangeable, the standard for clinical documents must support semantic interoperability [3]. Around the world, organizations mobilize efforts to develop templates to collect essential data to advance true data sharing, at least when systems adopt the same criteria. However, beyond the electronic medical records (EMR) proposal, the lack of customization for different clinical scenarios compromises the continuity of care based on connected systems, and the solution requires dedication from domain specialists [3].

Looking specifically at maternal and child health realities, the importance of dedicated data models for longitudinal communication during pregnancy is even more evident, since timely access to prenatal care data is a determinant of gestational and perinatal outcomes [7]. According to the World Health Organization (WHO), continuity of assistance is a critical line of action for reducing maternal and neonatal mortality [8]. During birth, women and neonates are more vulnerable to obstetric complications, and the existing information gap between prenatal consultations and hospital admissions contributes to the unacceptable rates of deaths and morbidities associated with pregnancy $[8,9]$. Adopting appropriate and timely prenatal practices can save lives and contribute to a positive pregnancy experience by allowing the birth of a healthy child without an unfavorable impact on maternal health [10]. In this scene, risk identification enables the prevention and appropriate treatment of complications, and anticipates the need for more complex care during childbirth and postpartum assistance [11].

This study aimed to develop a model for essential clinical records on prenatal care to be employed as reusable data between prenatal assistance and emergency care, sustained by health information systems. The primary hypothesis was that structural and semantic standardization can offer qualified obstetric information for health professionals in maternities. 


\section{Methods}

\section{Study design}

The present study comprised an applied and exploratory research of experts opinions on simulated scenarios of obstetric emergency caring, a minimum dataset proposal, and retrospective real scenario validation to achieve an information model for CCR.

\section{Settings, participants, and data sources}

The research protocol was approved by the institutional review boards in Brazil, the Ethics Committees of the Universidade Federal de Minas Gerais (UFMG), CAAE number 50171015.8.000.5149. The study took place in the Clinical Hospital UFMG/Ebserh, a public referral setting for high-risk pregnancies in the perinatal network of the city. A group of 9 of $15(60 \%)$ invited domain specialists attended to the request. Six (67\%) were female, and $3(33 \%)$ were male. Seven (78\%) were doctors and professors at the university hospital. They had extensive experience in gestation care, averaging 24 years of clinical practice, including prenatal or obstetric hospital care. Written informed consent was obtained from each participant.

\section{Procedures}

The steps to develop the information model occurred as actions represented in Figure 1.

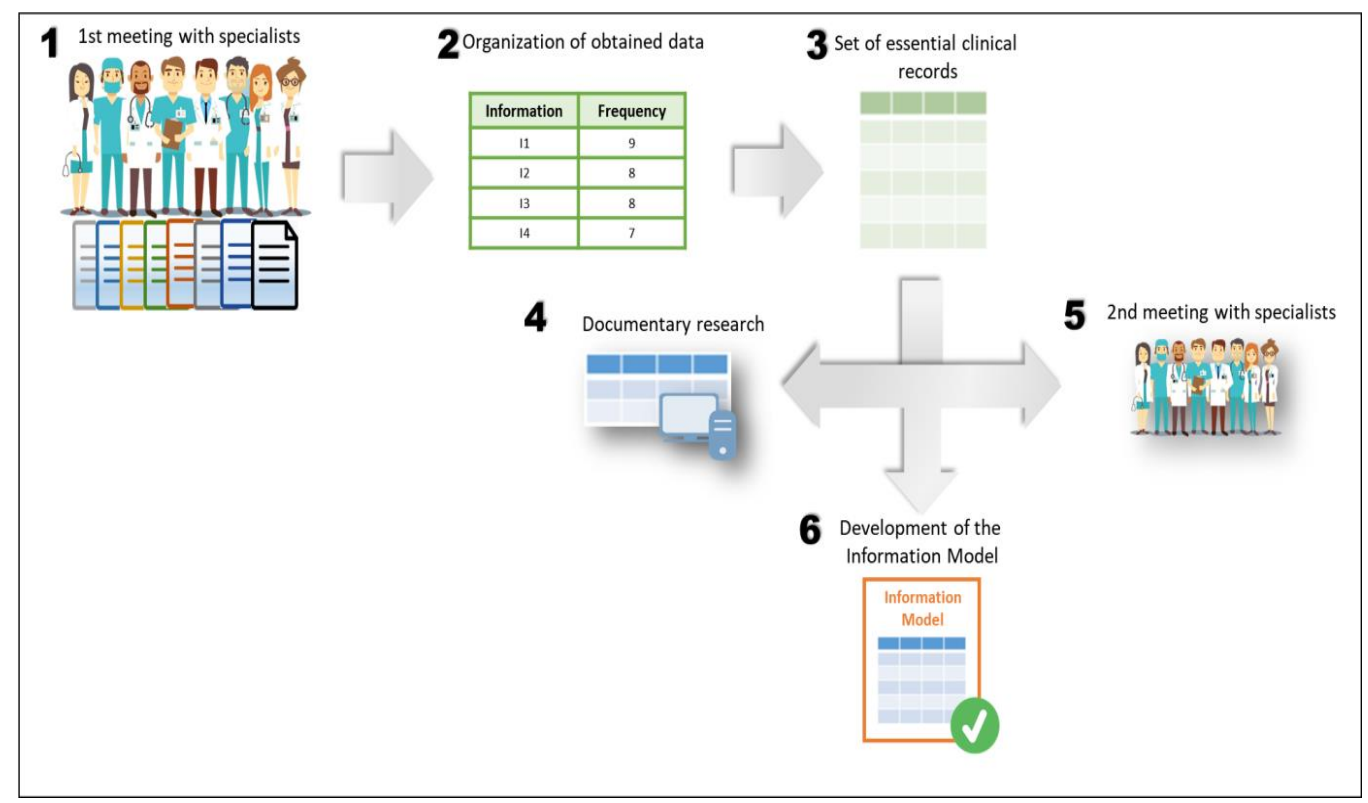

Figure 1. Procedures of the model for information development

The procedures tracked an interactive process between domain expert opinions, real EMR comparisons, and adjustments to obtain an information model useful for the practices required in obstetric admissions. The meeting with specialists consisted of surveying the most meaningful sets of data from prenatal care to support hospital maternity admission, according to their opinions (Step 1). A simulated informational setting was prepared by the authors with four storyboards picturing scenarios of pregnant women admissions in maternity hospitals described in Figure 2. 
Dear volunteer,

The following are four scenarios for you to place yourself in the context in which automated data exchange by electronic systems can be beneficial for the quality of care. In each one, think about what prenatal data you would like access to in order make the best decisions.

\section{History 1}

A pregnant woman arrives at the maternity hospital complaining of uterine contractions. She reports that she began prenatal care as soon as she got pregnant, underwent several consultations and exams, but recently lost her prenatal card. She denies any diseases, has no signs of clinical abnormalities and will be admitted for childbirth care. In your opinion, what are the most essential (prenatal) data that would support you to rate the gestational risk and provide the woman appropriate care for her immediate needs?

\section{History 2}

A pregnant woman arrives at the maternity hospital brought by SAMU (Mobile Emergency Care Service). She is in a post-coma state and her neighbor is her companion. You have a folder containing several results of laboratory tests, imaging, reports of inter-consultations with other specialists, prescription drugs, and her prenatal card. In your opinion, what are the most critical (prenatal) data that would support you to rate the gestational risk and provide the woman appropriate care for her immediate needs?

\section{History 3}

A pregnant woman arrives at the maternity clinic with fever, general malaise, and genital bleeding. She has already begun prenatal care and has had four appointments. She reports having had a cardiopathy prior to pregnancy. In your opinion, what are the most important (prenatal) data that would support you to rate the gestational risk and provide the woman appropriate care for her immediate needs?

\section{History 4}

You have just attended a prenatal appointment at a basic health unit. The pregnant woman brought an ultrasound result showing a morphological alteration of the nervous system (microcephaly) and a crooked foot. She has a history of a weekend visit to northeastern Brazil in early pregnancy. You suspect microcephaly by Zika virus. In your opinion, what are the most significant prenatal data that you would like to offer automatically to the hospital environment, in order to contribute to the continuity of care at the time of admission for delivery or any other hospital-based complications?

\section{Figure 2 - Storyboards picturing scenarios of pregnant women admissions in maternity hospitals.}

During the focus groups, each obstetrician individually analyzed the histories. Next, they were asked to write down the information they deemed essential for health professionals' decision making in such situations. Next, they were encouraged, in an open discussion with the other colleagues, to present a proposal for a minimum dataset to reuse information from the primary to the tertiary level of care, and facilitated by information systems. The authors of this paper did not interpose their contributions.

Next, a dataset was selected regarding their frequency of appearance in the speech and written consensus of the specialists (Steps 1 and 2). Data were grouped according to subject theme in the obstetric domain of knowledge (Step 3). Health informatics experts, with obstetrics background and the authors of this article, compared the proposed data with a sample of EMR registered during the hospital maternity admission, adjusting the modeling of data to the real informational scenario (Step 4). A 
second round with the specialists validated new elements and adjustments (Step 5). The selected set of data received a semantic standard, in line with international standard specifications $[12,13]$ (Step 6).

\section{Obstetric dataset validation}

Data modeling validation then proceeded to actual maternity EMR. The documentary analysis comprised 100 clinical histories randomly selected from 4,437 admissions, from January 8, 2015 to July 31, 2016. Ten (10\%) admissions were excluded because they did not represent viable pregnancies: $5(5 \%)$ of them reporting ectopic pregnancy, $3(3 \%)$ non-pregnant patients, and $2(2 \%)$ without any clinical content.

The informational EMR pathway was analyzed, as presented in Figure 3. First, women are identified in the reception of the emergency room. Next, a team of nurses screen the women according to their risk, using the Manchester protocol [14]. Obstetricians provide full clinical and obstetric evaluations, and decide whether the women should be admitted or discharged. The clinical EMR documentary record for this research comprised the initial assessments of the pregnant women recorded by obstetricians, which were retrieved from the database of the commercial EMR adopted in the hospital. The informational characteristics of this EMR were a small number of entries, and data-entry windows open for freewriting by the health professional.

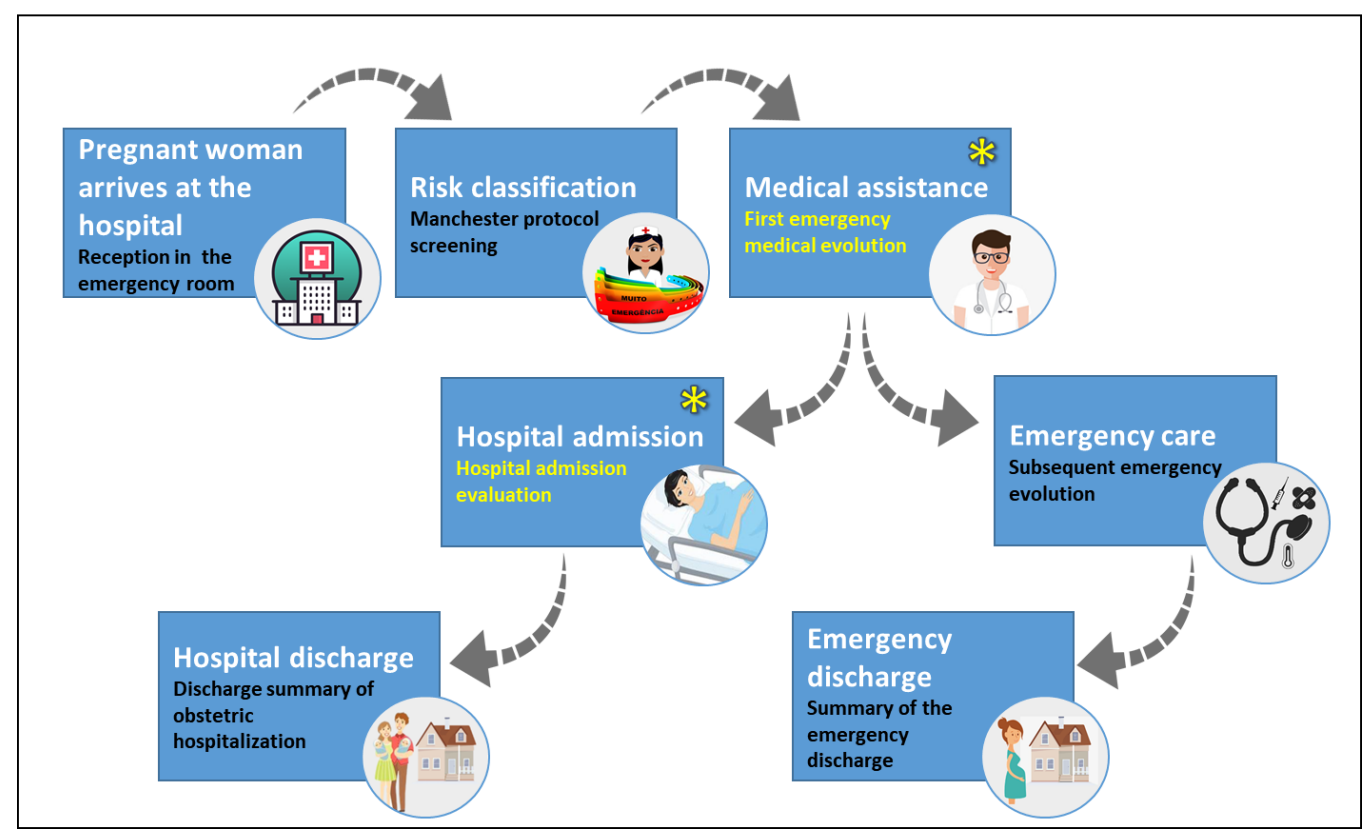

Figure 3. Service flow of pregnant women in maternity hospitals under emergency conditions.

* Indicates the clinical documents of the electronic medical records analyzed in the present study.

To process comparisons efficiently between the architecture of data from the proposal modeling with the real EMR, we developed an interface in PHP format with a PostgreSQL database. The authors analyzed 90 EMR histories and searched for data from the dataset proposed by obstetricians in the factual obstetric reports.

During the second round meeting with the domain specialists, adjustments from the validation analysis were discussed. The best practices were adopted to achieve 
consensus, together with the health interoperability documents published [15, 16]. New data records were proposed, and the existents validated, eliminated, or adjusted. Each data entry received a structured format, taking into consideration human-readable and ease of transferability between systems of information. At this point, restrictions, such as the type of data and the occurrence (single, multiple, mandatory, and optional) for each element were discussed.

\section{Modeling the architecture of the data}

Using the information model format, we specified the architecture of the data, and relationships between the information within each domain, as recommended by ABNT NBR 16472-1:2016 [12, 13]. This concept comprises the two-level modeling approach, adopted by openEHR and ISO 13606 standard specifications [15, 16], which proposes the use of an information model (or reference) to the generic representation of health information. The model of knowledge (or archetype) is the second level of modeling, in which domain-specific concepts are represented, based on terminologies and ontologies [17] which are not a target of this study.

\section{Results}

\section{Essential dataset, according to the obstetric specialists}

The set of data from prenatal care to be reused during admissions in the maternity hospital is presented in Table 1. The specialists unanimously suggested gestational age information. Checks for diseases and laboratory analyses were also priorities.

Table 1. Essential information from prenatal care for CCR in maternities, according to the obstetric specialist's opinions

\begin{tabular}{|l|c|c|}
\hline \multicolumn{1}{c|}{ Information } & \multicolumn{2}{c|}{ Frequency } \\
\cline { 2 - 3 } & $\mathbf{N}$ & $\mathbf{\%}$ \\
\hline Gestational age & 9 & 100 \\
\hline Laboratorial assessments & 8 & 88.9 \\
\hline Previous diseases & 7 & 77.8 \\
\hline Pregnant women age & 5 & 55.6 \\
\hline Obstetric history & 5 & 55.6 \\
\hline Complications related to the pregnancy & 4 & 44.4 \\
\hline Use of medicines & 3 & 33.3 \\
\hline Vaccines & 2 & 22.2 \\
\hline
\end{tabular}

\section{Validation of the dataset facing obstetric EMR}

Informal writing with extensive use of acronyms and abbreviations characterized unstructured reports in obstetric EMR. None of the 90 clinical reports utilized terminology to describe the diagnosis, laboratory assessments, or clinical examination. However, most of the re-useable data from prenatal care proposed by obstetric specialists were encountered in the real EMR. Obstetric history with the number of previous pregnancies was identified in 89 (98.9\%) records. The classification of pregnancy risk was informed in $58(92.1 \%)$ of the cases as "low-risk "or "high-risk," according to local practices and public policies to document the risk of complications or 
death [10]. The name of diseases or designation of obstetric risk conditions were reported in $63(70 \%)$ records. In $40(44.4 \%)$ clinical histories, one or more diagnoses of diseases were reported. Complementary check-tests assessments from the prenatal routine of care appeared in $61(67.8 \%)$ of the reports. Despite this, the dates of laboratory analyses were incomplete, many using only the day and month without specifying the year. Regarding antenatal markers of gestational age, the date of the last menstrual period was reported in $52(57.8 \%)$ of the electronic reports, though incomplete dates (missing day, month, or year) were also evident. The same missing information was observed for the date fields of the first obstetric ultrasound in 24 $(26.7 \%)$ reports.

The lack of standard when analyzing medical notes, even in digital free-writing, opened a window for the misinterpretation of null for missing data. For instance, the number of fetuses (single, double, triple, or more) was not explicitly described in 87 (96.7\%) documents, but were subsequently deemed single pregnancies based on the interpretation of other data, such as the result of an ultrasound indicating only one fetal weight. In 3 (3.3\%) medical reports, double gestation was indicated. In $30(33.3 \%)$, there was information regarding the partner of the pregnant woman. In $28(31.1 \%)$ reports, there where notes as to whether or not the gestation was planned . Religion was explicitly declared in 16 narratives $(17.7 \%)$. Information on smoking was verified in 40 (44.4\%), alcoholism appeared in 36 (40\%), and drug use in 12 (13.3\%). The birth plan, idealized by the pregnant women, was not stated in any records. However, the procedures for care, prepared by a physician, were indicated in $18(20 \%)$ reports, of which $6(33.3 \%)$ were due to clinical intercurrences in prenatal care, $4(22.2 \%)$ to elective cesarean programming, $4(22.2 \%)$ to labor induction, $2(11.1 \%)$ regarding glycemic control, and $2(11.1 \%)$ due to gestational age clarification.

Table 2 presents the adjustments carried out during the second meeting with the specialists. Vaccination was removed from the set of data as it was not mentioned in any EMR report, and it was considered to have no impact on the clinical decision in most emergency cases. Diseases and complications were arranged in the same section, named "Previous and current diagnoses." The identification of the active/inactive disease and date of diagnosis was added to better characterize these conditions of risk during the hospital stay. Entries for the registration of clinical, surgical, and therapeutic procedures were included to highlight previous surgeries and other treatments of the pregnant women, due to the extreme value placed on obstetric decisions, according to the domain specialists. Clinical-obstetric risk classification entries were inserted to indicate the presence of factors that point to some threat and require special attention.

Table 2. Essential information from prenatal care for CCR in maternities after adjustments

\begin{tabular}{|l|l|}
\hline Initial set of information & Final set of information \\
\hline Gestational age & Gestational age \\
\hline Laboratorial assessments & Laboratorial assessments \\
\hline Previous diseases & $\begin{array}{l}\text { Previous and current diagnoses, with of clinical, } \\
\text { surgical, and therapeutic details }\end{array}$ \\
\hline $\begin{array}{l}\text { Complications related to the } \\
\text { pregnancy }\end{array}$ & Pregnant women age \\
\hline Pregnant women age &
\end{tabular}




\begin{tabular}{|l|l|}
\hline Obstetric history & $\begin{array}{l}\text { Obstetric history, with clinical-obstetric risk factors } \\
\text { details }\end{array}$ \\
\hline Use of medicines & Use of medicines \\
\hline Vaccines & Removed \\
\hline & Social summary \\
\hline & Care Plan \\
\hline
\end{tabular}

The "Social Summary" section was included to record societal information that may impact hospital care, such as religion, schooling, use of illicit drugs, and domestic violence. During the second round discussions with domain specialists, ethical and cultural aspects were considered relevant. Two new sections were included in the dataset. The "Care plan" met the importance of indicating special needs during the assistance of pregnant women within high complexity facilities. To comply with a WHO recommendation [18], the element "Birth and emergency planning" was added to encourage the registration of the preferences of the pregnant woman [19].

\section{The information model}

The proposed information model, after adjustments of the validation step, was structured with nine sections, and fifty-six data entries (Online Resource available at http://www.techinfo.com.br/sbcas/Online_Resource.pdf). Column 1 describes the level of hierarchy for each entry. Column 2 presents the occurrence of information to represent the number of times that the element should appear. Column 3 describes the entry that exhibits the proposed element of information. Column 4 determines the type of data, characterizing the format and constraints.

\section{Discussion}

The main contribution of this study was the development of a generic information model dedicated to reusing prenatal care data in hospital settings. By gathering domain specialists' contributions, adjustments to the international standards for health data modeling, and real EMR validation, we expect to offer a readable data format for interface development in electronic health record (EHR) systems. The development of clinical document standards for semantic interoperability requires consensus on what data are really essential, chosen of their architecture, besides the hierarchical organization of entries in sections [20]. However, health professionals in the real scenario of care should recognize their practices of health assistance in the information model.

For communication between different levels of health assistance to be effective while using information systems requires a common language among providers, a thrifty use of acronyms while preserving patient safety [21]. Information technology has leveraged this communication on a worldwide scale, demanding standardized clinical documents to favor informational continuity [22]. The adoption of EHRs is regular practice in many scenarios, promoting collaborative work around health information technology to improve the efficiency of care [23]. Some of the global policies for health concern the promotion of the proper use of health information, protection against misinformation, supporting health management, and monitoring institutional performance by comparable health indicators $[2,24]$. 
Information obtained during pregnancy care is ample and detailed, as recommended in the guidelines for the best care [11]. Nevertheless, the present study was limited to a minimum set for data reuse in maternities. The Bipartisan Policy Committee reported that $70 \%$ of physicians consider a lack of interoperability as a crucial barrier to communication between professionals, and more than half of interviewees would like to receive only the information deemed essential to support clinical decisions [23]. In this sense, the availability of critical information on time may enable more qualitative hospital care [25].

We represented a number of severe clinical scenarios at the point of maternity admission using storyboards to guide the domain specialists during the first meeting. In all of them, the exchange of information, following a conceptual, terminological, and semantic pattern, had potential repercussions on the reduction of risks for women, lower institutional costs, and decreased the exposure of pregnant women to unnecessary examinations and procedures. However, the validation of the dataset comparing them with real EMR was crucial to obtain a set of information to represent the reality and finally to receive the architecture to be reused, while preserving semantics. We expect the gradual substitution of the free-writing information by terminologies or codified text will also contribute to the evolution of the information model.

Regarding the organization of the information model, the entries for diagnoses, examinations, and procedures were enabled in two modes, using terminology or free text. Similarly, the medication section due to the extensive variability range of drugs and their complementary data (dose, unit, etc.). Such flexibility in registries was based on previous experiences [13], justified by the still existing barrier regarding the use of terminologies. Concerning the birth plan, an element individualization of assistance, absent in all EMR, may represent minimum knowledge about its importance. This statement contains the women's preferences and promotes quality of care and the preservation of the rights of the pregnant woman [19].

Other reports have shown success in creating standardized clinical documents involving informatics and domain specialists. Medical forms structured as Multilingual Medical Data Models, based on interviews with physicians and health informatics specialists, had success in facilitating information sharing among practitioners in Germany [26]. An experience in China reported the development of reusable and standardized datasets from eight clinical documents, assisted by 25 professionals, including experienced physicians and health informatics specialists, to discuss the integrity, rationality, and accuracy of the reports [22]. Non-profit initiatives also foster the standardization of health information. The Brazilian Association of Technical Standards (ABNT), ISO in Brazil, holds periodic meetings with health and information technology professionals as well as government representatives, to discuss standards for health informatics. From this perspective, the "Discharge Summary for continuity of care - Part 1: Information Model" became a model for systems of information in the country [13]. The integration of archetypes of the discharge summary after childbirth and the government repository of data already has proof of concept in our systemic environment [6].

This study has limitations regarding the scope of the validation of the information model. Even though the methodology was based on experienced domain specialists' contributions and validated with EMR, external validation is limited. A 
proof of concept is required to adopt it. In addition, improvements and adjustments are necessary according to different scenarios, countries, and models of obstetric healthcare. Regarding the EMR analysis, the fragility of non-standardized and free-written reports was limited during the comparisons. It was possible to perceive that the terms may not be correctly understood and may undergo changes over time, and also differ according to the professional's specialty. Communication of technical information should be clear and unambiguous. Abuse of abbreviations is considered a critical barrier to understanding and the transparency of information [27]. The most significant problems related to abbreviations are different meanings, which have limited the use of abbreviations in medical records [21].

Finally, we believe that the development of an information model for the continuity of care records, based on a standard of semantic interoperability and useful for clinicians, has the potential to overcome the informality of EMR. The proposal contributes to a useful exchange of information between prenatal and emergency care to convert many local data into longitudinal information for many healthcare benefits.

\section{References}

[1] Kerber KJ, de Graft-Johnson JE, Bhutta ZA, Okong P, Starrs A, Lawn JE. Continuum of care for maternal, newborn, and child health: from slogan to service delivery. The Lancet [Internet]. Elsevier BV; 2007 Oct;370(9595):1358-69. Available from: http://dx.doi.org/10.1016/s0140-6736(07)61578-5

[2] Pan American Health Organization. eHealth in Latin America and the Caribbean: interoperability standards review. Washington, D.C.: PAHO 2016.

[3] Spyropoulos B, Botsivaly M, Tzavaras A, Koutsourakis K. Extending the use of DRGs to estimate mean Home-Care cost by employing an adapted ASTM E2369-05 Continuity of Care Record. 2006 International Conference of the IEEE Engineering in Medicine and Biology Society [Internet]. IEEE; 2006 Aug; Available from: http://dx.doi.org/10.1109/iembs.2006.260323

[4] Botsivaly M, Spyropoulos B, Koutsourakis K, Mertika K. Enhancing continuity in care: an implemantation of the ASTM E2369-05 Standard Specification for Continuity of Care Record in a homecare application. AMIA Annu Symp Proc. 2006;2006:66-70. PMID: 17238304; PMCID: PMC1839263.

[5] Adler-Milstein J, DesRoches CM, Kralovec P, Foster G, Worzala C, Charles D, et al. Electronic Health Record Adoption In US Hospitals: Progress Continues, But Challenges Persist. Health Affairs [Internet]. Health Affairs (Project Hope); 2015 Dec;34(12):2174-80. Available from: http://dx.doi.org/10.1377/hlthaff.2015.0992

[6] Santos MR, de Sá TQV, da Silva FE, dos Santos Junior MR, Maia TA, Reis ZSN. Health Information Exchange for Continuity of Maternal and Neonatal Care Supporting: A Proof-of-Concept Based on ISO Standard. Applied Clinical Informatics [Internet]. Georg Thieme Verlag KG; 2017;08(04):1082-94. Available from: http://dx.doi.org/10.4338/aci-2017-06-ra-0106

[7] Carroli G, Rooney C, Villar J. How effective is antenatal care in preventing maternal mortality and serious morbidity? An overview of the evidence. Paediatric and Perinatal Epidemiology [Internet]. Wiley; 2001 Jan;15(1):1-42. Available from: http://dx.doi.org/10.1046/j.1365-3016.2001.00001.x 
[8] Rai RK. Tracking women and children in a Continuum of Reproductive, Maternal, Newborn, and Child Healthcare (RMNCH) in India. Journal of Epidemiology and Global Health [Internet]. Atlantis Press; 2014 Sep;4(3):239-43. Available from: http://dx.doi.org/10.1016/j.jegh.2013.12.006

[9] Say L, Chou D, Gemmill A, Tunçalp Ö, Moller A-B, Daniels J, et al. Global causes of maternal death: a WHO systematic analysis. The Lancet Global Health [Internet]. Elsevier BV; 2014 Jun;2(6):e323-e333. Available from: http://dx.doi.org/10.1016/s2214-109x(14)70227-x

[10] Tunçalp Ö, Pena-Rosas J, Lawrie T, Bucagu M, Oladapo O, Portela A, et al. WHO recommendations on antenatal care for a positive pregnancy experience-going beyond survival. BJOG: An International Journal of Obstetrics \& Gynaecology [Internet]. Wiley; 2017 Mar 9;124(6):860-2. Available from: http://dx.doi.org/10.1111/1471-0528.14599

[11] Oladapo O, Tunçalp Ö, Bonet M, Lawrie T, Portela A, Downe S, et al. WHO model of intrapartum care for a positive childbirth experience: transforming care of women and babies for improved health and wellbeing. BJOG: An International Journal of Obstetrics \& Gynaecology [Internet]. Wiley; 2018 May 15;125(8):918-22. Available from: http://dx.doi.org/10.1111/1471-0528.15237

[12] Joint Initiative for Global Standards Harmonization Health Informatics Document Registry and Glossary. Standards Knowledge Management Tool 2014. Available from: http://www.skmtglossary.org/.

[13] ABNT. Health informatics - Discharge Summary for continuity of care Part 1: Information model. Brasilia: Associação Brasileira de Normas Técnicas (ABNT); 2016.

[14] Fakari FR, Simbar M, Modares SZ, Majd HA. Obstetric Triage Scales; a Narrative Review. Arch Acad Emerg Med. 2019 Jan 13;7(1):e13. PMID: 30847448; PMCID: PMC6377224.

[15] openEHR Foundation. openEHR Architecture Overview. BASE Release 1.1.0, 2018. Available from: https://specifications.openehr.org/releases/BASE/latest/architecture_overview.html

[16] International Organization for Standardization. Standard 13.606 Health informatics, Electronic health record communication. Part 1: Reference model. web: ISO; 2008. p. 108.

[17] Santos MR, Bax MP, Peçanha C, editors. Codificando Arquétipos em linguagem ADL com base no modelo de referência da norma ISO 13606. X Workshop de Informática Médica; 2010.

[18] World Health Organization. Birth and emergency preparedness in antenatal care. Intergrated Management of Pregnancy and Childbirth. [Internet]. Standards for Maternal and Neonatal Care, 2006. Available from: https://www.who.int/reproductivehealth/publications/maternal_perinatal_health/emer gency_preparedness_antenatal_care.pdf

[19] Beredugo L, Emmanuel L, Oniso J, Aluye-Benibo D. BIRTH PREPAREDNESS AND COMPLICATION READINESS IN AMASSOMA COMMUNITY OF 
SOUTHERN IJAW LOCAL GOVERNMENT AREA, BAYELSA STATE, NIGERIA. LAUTECH JOURNAL OF NURSING. 2018:160.

[20] Maia TA, De Muylder CF, Reis ZSN. Archetype Development Process: A Case Study of Support Interoperability Among Electronic Health Record in the State of Minas Gerais, Brazil. Trends and Advances in Information Systems and Technologies [Internet]. Springer International Publishing; 2018;457-67. Available from: http://dx.doi.org/10.1007/978-3-319-77703-0_45

[21] Kuhn IF. Abbreviations and acronyms in healthcare: when shorter isn't sweeter. Pediatr Nurs. 2007 Sep-Oct;33(5):392-8. PubMed PMID: 18041327

[22] Yang P, Pan F, Liu D, Xu Y, Wan Y, Tu H, et al. The Development of Clinical Document Standards for Semantic Interoperability in China. Healthcare Informatics Research [Internet]. The Korean Society of Medical Informatics (KAMJE); 2011;17(4):205. Available from: http://dx.doi.org/10.4258/hir.2011.17.4.205

[23] Bipartisan Policy Center. Clinician Perspectives on Electronic Health Information Sharing for Transitions of Care. 2012. Available from: https://bipartisanpolicy.org/wp-content/uploads/2019/03/Clinician-Survery_format2.pdf

[24] World Health Organization . WHO guideline: recommendations on digital interventions for health system strengthening. Geneva: World Health Organization; 2019. Licence: CC BY-NC-SA 3.0 IGO.

[25] Hawley G, Jackson C, Hepworth J, Wilkinson SA. Sharing of clinical data in a maternity setting: How do paper hand-held records and electronic health records compare for completeness? BMC Health Services Research [Internet]. Springer Science and Business Media LLC; 2014 Dec;14(1). Available from: http://dx.doi.org/10.1186/s12913-014-0650-x

[26] Kenneweg J, Fritz F, Bruland P, Doods D, Trinczek B, Dugas M, et al. Multilingual Medical Data Models in ODM Format. Applied Clinical Informatics [Internet]. Georg Thieme Verlag KG; 2012;03(03):276-89. Available from: http://dx.doi.org/10.4338/aci-2012-03-ra-0011

[27] Kushlan JA. Use and Abuse of Abbreviations in Technical Communication. Journal of Child Neurology [Internet]. SAGE Publications; 1995 Jan;10(1):1-3. Available from: http://dx.doi.org/10.1177/088307389501000101 